\title{
Structure and Electrical Transport of Single-Walled Carbon Nanotubes with Intramolecular Junction and Defects
}

\author{
Taekyung Kim and Jian-Min Zuo
}

\author{
Department of Materials Science and Engineering, Frederick Seitz Materials Research \\ Laboratory, University of Illinois at Urbana-Champaign, Urbana, IL, 61801, United States.
}

Since the discovery of carbon nanotubes (CNTs) in 1991, CNTs have attracted considerable scientific and technological interests because of their unique structural and electrical properties, the quasi 1D structure and ballistic electrical transport. While the structure of CNTs is remarkably close to ideal, straight, cylindrical tubes, defects such as 5-7 pairs still exist and a small curvature, or junction, can be introduced to the tube structure. The defects are important because they introduce local electronic states and/or change tube structure and consequently the tube's electronic properties. Here we present the structure analysis of a single-walled CNT (SWNT) with an intramolecular junction and its electrical transport measurements using an uniquely field-effect transistor device specifically designed for TEM [1]. Figure 1 shows a fabricated device with ultra thin metal catalyst films (Al/Fe/Mo of $8 \mathrm{~nm} / 1 \mathrm{~nm} / 0.5 \mathrm{~nm}$ thick) patterned on top of the Mo electrodes and a scanning micrograph of a SWNT across the electrodes. In order to determine the structure of the SWNT, we recorded diffraction patterns from the SWNT using nanoarea electron diffraction (NED) and compared with simulated diffraction patterns (DP) [2]. Figure 2(a) shows an example. The equatorial line of the CNT DP shown in Fig. 2(b) and the diffraction spots in Fig. 2(c) indicate that the CNT in this experiment is a chiral SWNT. Table 1 summarizes the experimental results. The average values of chiral angles and radii from 4 different DPs, which were taken from different parts of the SWNT. For the left half of the SWNT, the structure was determined to be $(28,17)$. The structure of the right half of the tube, however, required further analysis because the tube chiral angle and diameter ((30,13), $\alpha=17.14^{\circ} \mathrm{d}=14.90 \AA$ and (29.13), $\alpha=17.59^{\circ} \mathrm{d}=14.53 \AA$ by simulation) obtained from the experimental DP could be fitted with two possible structures within the error bar $\left(\alpha=17.57 \pm 0.1^{\circ}\right.$ and $d=14.83 \pm 0.2 \AA$ by experiment). Figure 3(a) displays the simulated DPs of two tubes of $(30,13)$ and $(29,13)$ structures. We found a very small difference in the s-DPs and compared them with e-DP in Fig. 3(b). It is found that s-DP of $(29,13)$ fits e-DP perfectly, on the other hand S-DP of $(30,13)$ shows a small discrepancy. Thus, electron diffraction results show that this particular tube has two different structures (both are semiconducting SWNTs), which are seamlessly fused together to form an intramolecular junction. The electrical transport characteristics of the tube were investigated by current-voltage $(I-V)$ measurements and found to be rectifying as shown in Fig. 4. This can be explained by the different band gaps of two different SWNTs. The mismatch in band-gaps results in an internal energy barrier as depicted in the insets of Fig. 4. Even though the exact structure the intramolecular junction is unclear from the DP, the benefits of identifying the structure of nanotubes is clear for understanding nanotube transport properties. The full potential of combining tube structural analysis using NED and transport property measurement will be demonstrated for investigating different types of defects in SWNTs.

[1] T. Kim, J. M. Zuo, E. A. Olson, I. Petrov, Appl. Phys. Lett. 87 (2005) 173108 
[2] M. Gao,J. M. Zuo, R. D. Twesten, I. Petrov, L. A. Nagahara, R. Zhang, Appl. Phys. Lett. 82 (2003) 2703

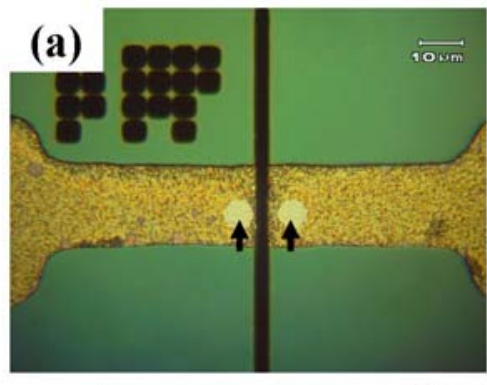

(b)

$1 \mu \mathrm{m}$

Figure 2 (a) Microfabricated device structure with a slit for TEM. Catalyst sites are black arrowed. (b) scanning micrograph of the SWNT bridging two electrodes.

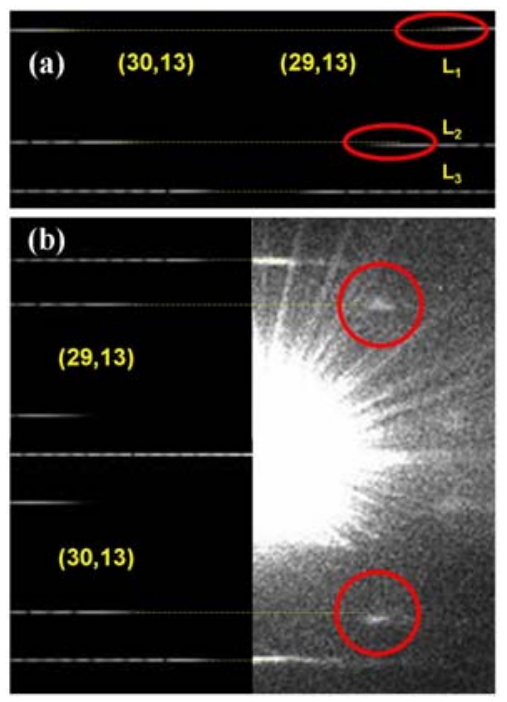

Figure 3 (a) Comparison of simulated DPs of the $(30,13)$ and $(29,13)$ SWNTs and the experimental DP.
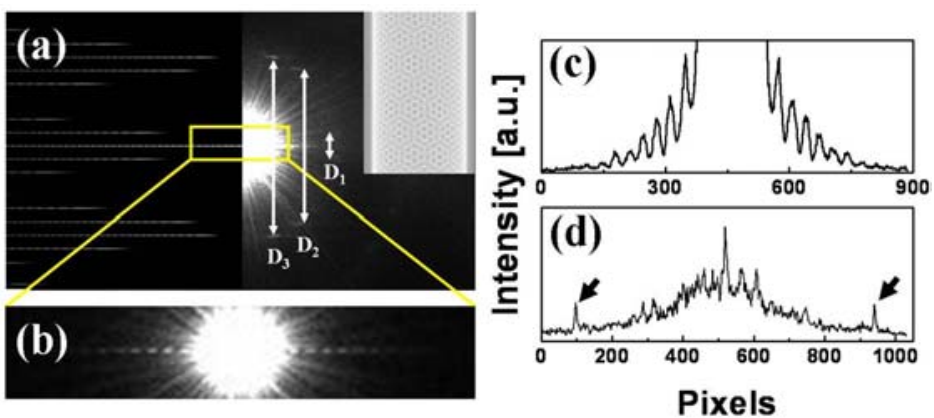

Figure 2 (a) Comparison of simulated (left) and experimental (right) DPs. Inset shows the structure model of the SWNT. (b) the equatorial line shows that the CNT is a SWNT. (c) and (d) display line profiles of the equatorial line and a diffraction spot, respectively.

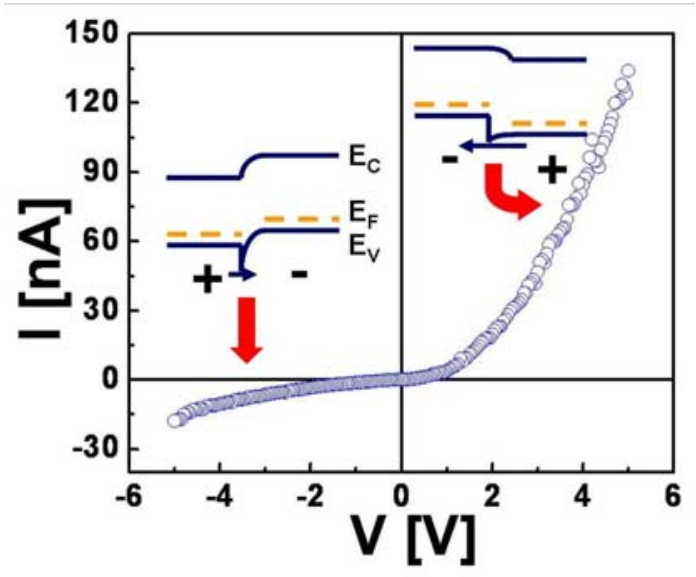

Figure 4 The I-V characteristic of the SWNT with an intramolecular junction. Insets show the schematic diagrams of the energy bands at the junction.

Table 1. Experimentally determined chiral angles and radii and possible fits from simulations

\begin{tabular}{cccc}
\hline & Chiral Angle & Radius & Possible Fits \\
\hline Left Half & $21.89^{\circ}$ & $15.35 \AA$ & $(28,17)$, semiconducting \\
Right Half & $17.57^{\circ}$ & $14.83 \AA$ & $(29,13),(30,13)$ semiconducting \\
\hline
\end{tabular}

